East African Medical Journal Vol. 85 No. 9 September 2008

GAUCHER'S DISEASE AT A NATIONAL REFERRAL HOSPITAL

F. Murila, MBChB, MMed (Paed), Department of Paediatrics and Child Health, J. A. Rajab, MBChB, MMed (Path.) and J. M. Ireri, HND, MLT, Department of Human Pathology, College of Health Sciences, University of Nairobi, P. O. Box 19676-00202, Nairobi, Kenya

Request for reprints to: Dr. J. A. Rajab, Department of Human Pathology, College of Health Sciences, University of Nairobi, P. O. Box 19676-00202, Nairobi, Kenya

\title{
GAUCHER'S DISEASE AT A NATIONAL REFERRAL HOSPITAL
}

\author{
F. MURILA, J. A. RAJAB and J. M. IRERI
}

\begin{abstract}
Objectives: To determine the prevalence and to characterise Gaucher's disease in terms of socio-demographic data, clinical presentation, and management as seen at Kenyatta National Hospital.

Design: A retrospective record based study.

Setting: Kenyatta National Hospital, a referral and teaching hospital.

Main outcome measures: Prevalence (number of cases seen a year), of Gaucher's disease, sociodemographic data, clinical presentation, mode of diagnosis and treatment modalities of Gauchers disease.

Results: Nine patients were studied, four males and five females giving a prevalence of 0.9 cases seen a year and a M:F ratio of about 1:1. The most common presentation was splenomegaly in nine $(100 \%)$ cases and hepatomegaly in seven $(78 \%)$ patients, neurological and bone symptoms were rare, in one $(11 \%)$ cases and in two $(22 \%)$ cases respectively. Diagnosis was mainly on basis of presence of Gaucher cells in bone marrow and splenic aspirate as enzyme assay was unavailable. Management was mainly supportive and enzyme therapy was only available for two $(22 \%)$ patients. Anaemia was the most common complication with seven $(78 \%)$ patients and one death occurred due to osteomyelitis. Only four $(44 \%)$ patients were followed up for a period of four years.

Conclusions: Gaucher's disease is a rare condition at the Kenyatta National Hospital (KNH). The presentation of most patients is organomegaly, (hepatosplenomegaly) and best fits the type 1 or non-neuronopathic Gaucher's disease. Neurological manifestations are rare. Management of this condition at the $\mathrm{KNH}$ is mainly supportive and enzyme therapy still remains out of reach for most patients.
\end{abstract}

\section{INTRODUCTION}

Gaucher's disease (GD) results from an inborn error of glycophospholipid metabolism caused by deficient activity of lysosomal hydrolase called glucocerebrosidase. The defect causes progressive accumulation of glycolipid substrates, particularly glycosylceramide in the reticuloendothelial cells. This accumulation results in infiltration of the bone marrow, hepatosplenomegaly and skeletal complications (1). The Gaucher's cell that characterises this disorder is of macrophage lineage. The genetic pattern is autosomal recessive and the sex distribution is equal (2).
Gaucher's disease is the most common lipid storage disorder and most common genetic disease in the Ashkenazi Jews with a prevalence of 1 per 1,000 . It is rare in the non-Jewish population with an estimated frequency of 1 in 40,000 . The overall frequency globally is about 1 in 200,000 and the disease has been reported in non-Jewish Caucasians as well as natives of Greece, India, China and Japan (3). The local prevalence is unknown although hospital records indicate that cases are seen in our setting. Similarly, data from other African countries is lacking.

Clinically three distinct types are seen and classified on the basis of the presence and 
progression of neurological complications. Type 1 or non-neuronopathic Gaucher's disease is the most common clinical variant of the disease. It is particularly prevalent in Ashkenazi Jews in whom it may affect up to 1 in 450 individuals. This may be the prevalent clinical variant in our setting and studies are currently in progress to elucidate the nature and severity of symptoms in patients with mild disease. Type 2, or acute neuronopathic Gaucher's disease, is a rare, pan-ethnic variant that characteristically presents in early infancy (average age three months) with obvious severe CNS involvement (e.g. Seizures, trismus, strabismus, psychomotor retardation, spasticity, sucking and swallowing difficulties) with hepatosplenomegaly. It progresses rapidly to death before the age of two to three years and has no ethnic predilection. Type 3, or subacute neuronopathic Gaucher's disease, classically presents in middle childhood as a progressive neurodegenerative disorder (type 3a); however, it may also present with severe, ultimately fatal, visceral disease.

The clinical presentation of this disorder has not been studied in our setting. Owing to its non-specific presentation, (hepatosplenomegaly which is common in many other tropical conditions) diagnosis may be missed due to the low index of suspicion. This disease is amenable to treatment albeit costly and enzyme replacement has been shown to be effective and safe. Hospital records document one to two cases seen annually in the paediatric referral wards.

The measurement of glucocerebrosidase activity in peripheral blood leukocytes, skin fibroblasts, amniocytes, or chorionic villi remains the most universally effective method in the diagnosis of GD with heterozygotes having approximately one half of the normal enzyme activity. The presence of Gaucher's cells in the bones and liver biopsy is almost always pathognomonic of GD $(1,4,5)$. Molecular diagnosis is feasible in some circumstances. In our setting, clinical symptomatology and the presence of Gaucher's cells in bone marrow or spleen are the mainstay of diagnosis. Enzyme assays are not available locally.

Treatment with an acid beta glucosidase prepared with recombinant DNA technology or obtained from the placenta offers considerable clinical improvement with enhanced quality of life (3). This therapy decreases liver and spleen size, reduces some skeletal abnormalities, and successfully reverses other manifestations of the disorder including abnormal blood counts. Bone marrow transplantation has cured a small number of patients and steroids have been reported to alleviate symptomatology during crisis $(6,7)$. Splenectomy may be performed for thrombocytopenia (4). Future treatment may include gene therapy.

\section{MATERIALS AND METHODS}

Case notes of all patients diagnosed with Gaucher's disease on basis of presence of Gaucher's cells in bone marrow or splenic aspirates between the period of January 1995 and December 2005 were examined and relevant data (socio-demographic, presentation, mode of diagnosis, management modalities and outcome) were collated on to a structured form and analysed.

\section{RESULTS}

There were nine cases of Gaucher's disease of whom four $(44 \%)$ were male and five $(56 \%)$ were female giving a male to female ratio of 1:1.25. The ages ranged from four months to seven years with a mean of four years. Of the patients seen six $(67 \%)$ hailed from the Eastern province of Kenya and four (44\%) from the Central province.

The majority, six $(67 \%)$ were of Kikuyu ethnicity while the remaining three $(33 \%)$ were of Embu, Meru and Kamba ethnicity. At the time of diagnosis, symptoms had been present for a duration of one month to two years.

The age at presentation ranged from one year three months to six years with a mean of four years three months. The clinical features were as follows in order of frequency; splenomegaly in nine (100\%), hepatomegaly in seven (78\%), failure to thrive seven $(78 \%)$, pallor seven $(78 \%)$, lymphadenopathy six $(67 \%)$, fever four (44\%), bleeding two (22\%) and bone fracture two $(22 \%)$. Lethargy, neurological symptoms and oedema alloccurred only in one $(11 \%)$ patient each and jaundice and bone pain were not among the presenting clinical features. The neurological presentation was in the form of delayed milestones in a patient with failure to thrive who was at the time seven years old. Spleen enlargement ranged from $6-24 \mathrm{~cm}$ while the liver enlargement was smaller $4-11 \mathrm{~cm}$ (Table 1).

Table 1

Clinical features of Gaucher's disease

\begin{tabular}{lcc}
\hline Clinical feature & No. & $(\%)$ \\
\hline Organomegaly & & \\
$\quad$ Spleen & 9 & 100 \\
Liver & 7 & 78 \\
$\quad$ Lymphadenopathy & 6 & 67 \\
Failure to thrive & 7 & 78 \\
Infections & 5 & 55 \\
Bleeding & 3 & 33 \\
Bone & & \\
$\quad$ Pain & 0 & 0 \\
$\quad$ Fracture & 2 & 22 \\
$\quad$ Osteoporosis & 1 & 11 \\
$\quad$ CNS & 1 & 11 \\
\hline
\end{tabular}


The diagnosis of Gaucher's disease was made on bone marrow aspirate in eight $(89 \%)$ of the cases and one $(11 \%)$ on splenic aspirate. No patient had enzyme assays performed for confirmation of the diagnosis. Enzyme therapy was administered in only two $(22 \%)$ of the patients. The other patients received only supportive care which consisted of red blood cells, fresh frozen plasma and platelet transfusions, antibiotics, anti-tuberculosis treatment, analgesics, and haematinics. Traction and plaster of paris application were performed in the two patients with fracture neck of femur and fracture femur respectively.

In the haematological findings, seven $(78 \%)$ had anaemia (haemoglobin 4 to $10.9 \mathrm{~g} / \mathrm{dl}), 3(33 \%)$ had thrombocytopaenia (platelets 94 to $\left.149 \times 10^{9} / \mathrm{L}\right)$ and only one (11\%) had leucopaenia (WBC $2 \times 10^{9} / \mathrm{L}$ ). Only one $(11 \%)$ patient had osteoporosis. The liver enzymes were normal in all nine $(100 \%)$ patients but two $(22 \%)$ had a raised International Normalised Ratio (INR) (Table 2).

Table 2

Laboratory features of Gaucher's disease

\begin{tabular}{lll}
\hline \multicolumn{1}{c}{ Laboratory feature } & No. & $(\%)$ \\
\hline Total Blood Count & & \\
$\quad$ Anaemia & 7 & 78 \\
Thrombocytopenia & 4 & 44 \\
$\quad$ Leucopenia & 1 & 11 \\
Radiological imaging & & \\
$\quad$ Fracture & 2 & 22 \\
$\quad$ Osteoporosis & 1 & 11 \\
$\quad$ Respiratory infection & 1 & 11 \\
Raised acid phosphatase & 3 & 33 \\
\hline
\end{tabular}

There was one death which was secondary to osteomyelitis. Only four $(44 \%)$ of the patients were followed up and this was for a duration of two to four years.

\section{DISCUSSION}

Gaucher's disease has been divided into three main types based on the clinical presentation. Most of the patients $(89 \%)$ did not have neurological symptoms and had presented at the age of one year and beyond. These most likely had type 1 disease which is by far the most common variant of the disease and is particularly prevalent among the Ashkenazi Jews $(6,7)$.There was only one patient aged seven years with neurological symptoms which had manifested as delayed milestones of infancy. This patient most likely had type 2, the neuropathic disease which presents in early infancy $(8,9)$.

Gaucher's disease is inherited as an autosomal recessive trait and males and females are equally affected (2). This was illustrated in this study where the male to female ratio was 1:1.25. Our patients were all either from the Eastern province $(67 \%)$ or the Central province and the majority $(67 \%)$ were of Kikuyu ethnicity. This is most likely due to the location of the national referral hospital which is within easy reach of the Eastern and Central provinces where these ethnic groups are found. However, the possibility of a genetic predisposition in this group cannot be excluded and there is need for further genetic studies in this population.

Confirmation of Gaucher's disease is by demonstration of reduced levels of the enzyme glucocerebrosidase, however, this technique is still unavailable in the hospital. Diagnosis, therefore, rested on the finding of the Gaucher's cell in either the bone marrow or the spleen. Gaucher's - like cells may occur in other conditions primarily neoplastic or haematologic disorders associated with increased red cell or leucocytes turnover like leukaemia especially chronic granulocytic leukaemia and hairy cell leukaemia, Hodgkin's, Non Hodgkin's disease, lymphoma. These cells are also found in thallasaemia and acquired immunodeficiency syndrome (10). Most of these conditions present with a clinical picture that is similar to that of Gaucher's disease with splenomegaly, hepatomegaly, fever, lethargy, anaemia and bleeding.

In all three types of the disease, splenomegaly is usually the earliest sign and is often complicated by hypersplenism leading to thrombocytopaenia with bleeding diasthesis, anaemia and leucopaenia. Hepatomegaly is another frequent sign of Gaucher's disease but is usually milder than the splenomegaly. All our patients had splenomegaly as a feature while 78\% had hepatomegaly. The hepatomegaly (range $4-12 \mathrm{~cm}$ ) was typically milder than the splenomegaly (range 6-24cm) (11).

Zimran et al (12) studied 53 patients who were mostly in their early adulthood with a mean age of 33 years. He found that the most common presenting symptom was bleeding related to splenomegaly and thrombocytopaenia and that the chronic symptoms evaluated for over a period of 20 years after the diagnosis were mainly skeletal.

Haematologic abnormalities, a consequence of splenic sequestration and over activity of Gaucher's cell infiltration of the bone marrow and displacement of haematopoetic elements, comprise some of the most common problems in Gaucher's disease $(11,13)$. Mild to severe thrombocytopaenia $(<20,000 / \mu \mathrm{L})$ and normocytic, normochromic anaemia are the most frequent such abnormalities. Erythrocyte and placelet survival is decreased, and coagulopathy and haemorrhagic diathesis may develop (13).

In this study seven $(78 \%)$ had anaemia four $(44 \%)$ had thrombocytopaenia and only one $(11 \%)$ had leucopaenia. Bleeding was rare and was seen only in two patients $(22 \%)$. 
Skeletal disease in Gaucher's disease is common occurring in $50-100 \%$ of cases $(11,14,15)$. The aetiology is not fully known, but a key pathogenetic process appears to be the progressive infiltration and replacement of bone marrow trabecular bone by Gaucher's cells. Skeletal complications can be divided into five major categories: failure of remodeling, osteopaenia, osteonecrosis leading to fractures, osteosclerosis, and bone crises, acute episodes of excruciating pain resembling those in sickle cell anaemia (16). Skeletal disease in our population was less common than in the reported series, 30\%. Children and adolescents with Gaucher's disease sometimes suffer growth stunting and a pubertal delay as was seen in our series where seven $(78 \%)$ were underweight. Our patients were evaluated in a referral centre and therefore the data obtained needs to be interpreted with great caution when applying to the general population which most probably contains a greater population of very mild cases. Enzyme replacement therapy for type one disease has been available for the last ten years and is known to result in clinical improvement with enhanced quality of life, dramatic reduction of hepatosplenomegaly within one to three years and resolution of both the haematologic and splenic derangements $(3,17-20)$. In our study only two $(22 \%)$ of the patients were started on enzyme replacement therapy due to its prohibitive cost. The two, a girl and her sibling, were at the time spending 800USD and 400USD respectively per month for this treatment.

In conclussion, Gaucher's's disease is a rare disease in paediatric practice at the Kenyatta National Teaching and Referral Hospital. The most common presentation is hepatosplenomegaly. With most patients falling in the category of type I Gaucher's disease. Neurological and skeletal disease appear to be a rare presentation in our population. Enzyme replacement therapy still remains out of reach for most patients.

\section{REFERENCES}

1. Cox, T.M. Gaucher's disease: understanding the molecular pathogenesis of sphingolipidoses. J. Inherit. Metab. Dis. 2001; 24 (Suppl 2): 106.

2. Gabrowski, G. A. Genetic aspects of Gaucher. Gaucher Clin. Perspect. 1992; 1: 5-9.

3. Gabrowski, G.A. Gaucher's disease: Lessons from a decade of therapy. J. Pediatr. 2000; 144: 515-519.

4. Brady, R.O., Kanfer, J. and Shapiro, D. The metabolism of glucocerebrosides, I. Purification and properties of a glucocerebroside-cleaving enzyme deficiency: use of purified glucocerebrosidase in Gaucher's disease. N. Engl J. Med. 1974; 291: 989-993.
5. Brady, R.O., Barton, N.W. and Grabowski, G.A. The role of neurogenetics in Gaucher's disease. Arch. Neurol. 1993; 50: 1212-1224.

6. Beutler, E. Gaucher's disease. N. Engl. J. Med. 1991; 325:1354-1360.

7. Rice, E. O., Mifflin, T. E., Sakallah, S. and Lee, R. E. Gaucher's disease: Studies of phenotype. Molecular diagnosis and treatment. Clin. Genet. 1996; 49:111.

8. Patterson, M.C.,Horowitz, M., Abel, R.B., etal.Isolated horizontal supranuclear gaze palsy as a marker of severe systemic involvement in Gaucher's disease. Neurology. 1993; 43: 1993-1997.

9. Cox, T. M. and Schofield, J. P. Gaucher's disease: clinical features and natural history. Baillieres Clin. Haematol. 1997; 10: 657-689.

10. Barranger, J. A. and Rice, O.E. Gaucher's disease: Diagnosis monitoring, and management. Molecular Med. Therepeut. 1997; 5: 1.

11. Barranger, T.A. and Rice, E. An overview of Gaucher's Disease. Gaucher Clin. Perspect 1993;1: 1-5.

12. Zimran, A., Kay, A., Gelbart, T., Garver, P., Thurston, D. Saven, A. and Beutler, E. Medicine (Baltimore). 1992; 71: 337-353.

13. Esplin, J.A. The diagnosis of Gaucher's disease in the hematologicimpact of Gaucher's disease: proceedings of a symposium, Dec $4^{\text {th }} 1992$, Anaheim, CA. Pawling, NV, Caduceus medical. 1993; 8-9.

14. Barranger, J.A. and Ginns, E.L. Glucosylceramide lipidoses; Gaucher's disease, in Scriver CR (ed.): The Metabolic Basis of Inherited disease (ed 6.). New York, NY, MGraw-Hill, 1989; pp 1677-1698.

15. Beighton, P., Goldlat, J. and Sacks, S. Bone involvement in Gaucher's disease, in Desnick, R.J., Gatt, S. and Grabowski, G.A. (eds): Gaucher's Disease: A century of delineation and research. Proceedings of the fist international symposium on Gaucher disease, New York, 1981. New York, 981, N, Liss, 1982; pp 107-129.

16. Einhorn, T.A. Managing orthopaedic manifestations of Gaucher's disease. Pawling, NY.Caduceaus Medical, 1993; pp 1-14.

17. Barton, N.W., Furbish, F.S., Murray, G.J., and Garfield, M. Therapeutic response to intravenous infusions of glucocerebrosidase in a patient with Gaucher's disease. Proc. Natl. Acad. Sci. USA. 1990; 87:1913.

18. Barton, N.W., Brady, R.O., Dambrosia, J.M., D.J. and Bisceglie, A.M. Replacement therapy for inherited enzyme deficiency - macrophage-targeted glucocerebrosidase for Gaucher's disease. N. Engl. J. Med. 1991; 324:1464.

19. Friedman, B., Vaddi, K., Preston and C. Mahon, E. A comparison of the pharmacological properties of carbohydrate remodeled recombinant and placental derived beta-glucocerebrosidase: implications for clinical efficacy in treatment of Gaucher's disease. Blood. 1999; 93: 2807.

20. Weinreb, N.J., Charrow, J., Anderson., et al. Effectiveness of enzyme replacement therapy in 1028 patients with type 1 Gaucher's disease after 2 to 5 years of treatment: a report from the Gaucher registry. Am. J. Med. 2002; 113: 112-119. 